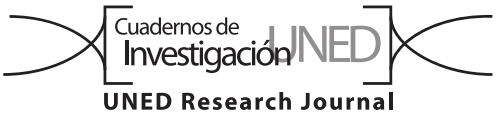

\title{
La electrificación del transporte público: retos y oportunidades para Costa Rica
}

\author{
Verena Arauz Schulze-Boysen \\ Grütter Consulting, Thirsteinerstr.22 4153, Reinach, Suiza; verena.arauz@googlemail.com
}

Recibido 10-IX-2018 • Corregido 16-XII-2018 • Aceptado 14-I-2019

\begin{abstract}
The transport sector is the main contributor of greenhouse gases in Costa Rica. Air quality in this country surpasses levels accepted by the World Health Organization. A mitigation effort in regards to these gases is sustainable transportation, with a significant reduction in emissions by this sector. Costa Rica is currently moving towards a zero-emission automobile fleet, with electric cars. In this article I discuss the possibilities for Costa Rica to electrify its public bus fleet, in particular electric buses and hydrogen cells, and compare it with other international experiences.
\end{abstract}

Key words: Climate change, mitigation, greenhouse gases, sustainable transportation.
RESUMEN: The electrification of public transport: challenges and opportunities for Costa Rica. El sector transporte es el principal contribuyente de los gases de efecto invernadero en Costa Rica. La calidad del aire en este país sobrepasa los niveles aceptados por la Organización Mundial de la Salud. Una iniciativa hacia la mitigación de dichos gases es el transporte sostenible, con reducción significativa en emisiones en este sector. Costa Rica actualmente está moviéndose hacia una flota vehicular de cero emisiones, con automóviles eléctricos. En este artículo se analizan las posibilidades de Costa Rica para electrificar su flota de autobuses para el transporte público, en particular buses eléctricos y de celdas de hidrógeno y se compara con otras experiencias internacionales.

Palabras Clave: Cambio climático, mitigación, gases de efecto invernadero, transporte sostenible.
El sector transporte en Costa Rica no solo representa alrededor de un 38\% de los Gases de Efecto Invernadero, pero más allá, los niveles de material particulado en la atmósfera también son alarmantes. Según el último informe sobre la calidad del aire, PM 10 y sobretodo PM 2.5 sobrepasan las directrices dictadas por la Organización Mundial de la Salud significativamente. Para solventar estos problemas, en países desarrollados se han implementado estrictos estándares de emisión para vehículos. En los últimos años, la misma industria automotriz, ha alterado el software de los vehículos, para arrojar valores de emisión menores que los que se requieren. El hecho de que se haya recurrido a estos métodos, es una prueba de lo rigurosas que deben ser este tipo de mediciones.

Mientras tanto, en Costa Rica circulan vehículos de más de 50 años; los camiones cisterna tienen una edad promedio de 25 años (Riteve, 2017). Si bien el país no siguió las tendencias a nivel mundial de reducir la contaminación causada por los vehículos en las ciudades, siendo más rigurosos y restrictivos con estándares de emisión, ahora está a las puertas de poder dar un salto promoviendo vehículos de cero emisiones de escape es decir $100 \%$ eléctricos.

Con algunas estaciones de recarga públicas en diferentes áreas del país y una exención de impuestos para algunos vehículos eléctricos, se tomaron pasos en la dirección correcta. Queda que el individuo tome la decisión de cambiar su carro por uno eléctrico, y esa transición ha sido lenta. Las condiciones para los vehículos comerciales, aún tienen potencial para ser mejoradas. En este artículo se hablará de las posibilidades que tiene en particular Costa Rica para electrificar su flota de autobuses para el transporte público. 


\section{ALTERNATIVAS TECNOLÓGICAS EN DISCUSIÓN PARA REDUCIR LAS EMISIONES DE LOS BUSES EN COSTA RICA}

Para reducir las emisiones, tanto de contaminantes locales como de gases de efecto invernadero, en el transporte público, se han abordado diferentes alternativas. Alternativas como Gas Natural Licuado o Comprimido, si bien tienen un impacto en la reducción de contaminantes, no serán tratadas en este artículo, ya que sigue siendo un combustible fósil. En un país que genera el $98 \%$ de su electricidad con fuentes renovables como Costa Rica, a alternativa de la electrificación es la más viable. También se abordará el hidrogeno como fuente de energía, ya que esta tecnología también ha recibido cierta atención en los últimos meses. Como fuente principal, se toma el informe elaborado en Febrero del 2018 por la firma Grütter Consulting, por encargo del Banco Interamericano de Desarrollo "Tecnologías alternas para buses en Costa Rica".

\section{BUSES ELÉCTRICOS}

Los buses eléctricos (Battery Electric Bus- BEB) son vehículos con motores eléctricos y baterías que se cargan en la ruta o que se cargan en sitios específicos durante el día o la noche. Las principales diferencias entre los distintos tipos de BEBs están relacionadas con el sistema de carga de la batería, la flexibilidad operativa, el peso del vehículo y la capacidad de pasajeros, así como en el costo del vehículo y de la infraestructura.

Es importante saber diferenciar entre los diferentes sistemas de carga, ya que de eso dependería la viabilidad para electrificar las diferentes rutas de autobús. Un bus de carga lenta durante la noche necesita ir equipado con una gran cantidad de baterías para asegurar un rango diario suficiente para cubrir todas las actividades. Esto implica un gran requerimiento de espacio y un alto peso debido a las baterías, así como un complejo sistema de gestión de las baterías. En un bus de $12 \mathrm{~m}$ las capacidades típicas de la batería son del orden de 350-450kWh para permitir un rango de operación de $200-300 \mathrm{Km}$.

Los buses con posibilidad de carga rápida han aumentado mucho en popularidad debido a la disponibilidad y precios más bajos de cargadores con una potencia nominal de 300-500kW. Los buses se pueden cargar en 15-30 minutos dependiendo de la capacidad de la batería del bus, la unidad de carga y el número de tomas de corriente del bus (en general 2 y en algunos modelos 4). La capacidad de la batería de estos buses es de 100-200kWh y en general se cargan una vez durante el día con carga rápida y adicionalmente en la noche con carga lenta.

Los sistemas de "carga de oportunidad" se caracterizan por operar con buses híbridos enchufables o con buses eléctricos que se cargan en ruta, ya sea en puntos de recarga en todo el circuito del bus o en la primera $\mathrm{y} / \mathrm{o}$ última parada. Estos sistemas se utilizan principalmente para buses de alta capacidad (más de $12 \mathrm{~m}$ de largo) $y$ en vista de que se requiere una infraestructura de recarga a lo largo de la ruta, es preferible que se tenga un carril segregado. Otra alternativa para buses de alta capacidad, que ha resurgido en los últimos años, son los trolebuses. Hoy en día, vienen equipados con baterías, que les permite un rango de autonomía (sin catenaria) de hasta $30 \mathrm{Km}$.

\section{BUSES DE CELDAS DE HIDRÓGENO}

Una celda de combustible es esencialmente una batería primaria de alimentación continua. Al igual que las baterías convencionales, las celdas de combustible son dispositivos electroquímicos que generan electricidad directamente por separación de cargas positivas y negativas. Un bus pila de combustible de hidrógeno utiliza este gaas como fuente de energía para las ruedas de accionamiento, a veces aumentada con baterías o un super-capacitador.

El rango de operación de buses a hidrógeno es de $300 \mathrm{Km}$, lo cual es comparable con buses convencionales, mientras que el tiempo de carga es de aproximadamente 3-5 minutos (Gobierno Londres, 2017). Esto les da una significativa ventaja en comparación con los BEB.

Actualmente, la tecnología está siendo probada en flotas piloto de varias ciudades del mundo; sin embargo, no hay aún experiencias con una flota de un tamaño representativo. Todavía se trata de una tecnología experimental, y los esfuerzos de investigación siguen siendo importantes, pero no es recomendable aplicar esta tecnología actualmente para un servicio que no se puede dar el lujo de tener pérdidas en cuanto a la calidad. Además, al ser una tecnología en desarrollo, el precio del bus puede ser de alrededor de 800000 USD, no hay datos exactos, porque no se fabrican en serie aún.

\section{EXPERIENCIAS INTERNACIONALES}

La flota más grande de autobuses eléctricos se encuentra en la República Popular de China. Según el estudio elaborado por el Banco Asiático de Desarrollo (ADB), Sustainable Transport Solutions - Low Carbon Buses in 
the People's Republic of China, para finales del 2017 en este país circulaban alrededor de 380000 buses eléctricos o híbridos enchufables. Este rápido incremento se dio, gracias a subsidios importantes por parte del gobierno central y los gobiernos locales. Sin embargo, muchas ciudades aún encuentran retos para poder electrificar su flota al $100 \%$, sobre todo para rutas más largas y buses más grandes.

Si bien los subsidios son un mecanismo importante para facilitar la transición y así también descontaminar el aire de las ciudades, muchas empresas de autobús no logran optimizar la distribución de las baterías, resultando así, en operaciones deficientes. La transición debe ser asistida rigurosamente por la investigación y técnicos capacitados.

\section{OPCIONES PARA COSTA RICA}

En vista de que los buses 100\% eléctricos ya han sido ampliamente probados en otros países y que hay disponibilidad de datos, la opción más recomendable descarbonizar el transporte público es, pasarse a esta tecnología. Sin embargo, es menester hacer cálculos rigurosos para asegurar el éxito.

La ley que incentiva los vehículos eléctricos, exige que cada dos años, las empresas deben sustituir un $5 \%$ de su flota de buses de diésel por eléctricos. Sin embargo, hay rutas que son muy largas y la tecnología no da para cubrir ese rango.

Un aspecto importante es que BEBs tienen en general una menor capacidad de carga de pasajeros por lo cual se requiere de una mayor cantidad de buses en épocas pico para poder mantener el mismo nivel de servicio. Por sus limitaciones de rango y de potencia se presta más para un uso en rutas cortas, con menor afluencia de personas y que sean relativamente planas.

Si se analizan las diferentes rutas dentro del GAM, se puede concluir que las carreras (un viaje ida y vuelta) rara vez sobrepasan los 30Km (ARESEP, 2018). Además, si bien hay rutas con pendientes de hasta un $50 \%$ y se clasifican como montanas, la mayoría son relativamente planas. Dadas esas condiciones, la mejor tecnología alterna disponible y adecuada para la GAM serían BEBs con una cantidad de baterías moderada (aprox. 200kWh) realizando una carga rápida diurna y una carga lenta nocturna.

Otro factor que beneficia la transición a esta tecnología, es el precio relativamente bajo de la electricidad. (Sobre todo en horas valle, que es cuando se cargarían los buses). Esto conlleva a costos totales en la vida útil comercial (total cost of ownership, TCO) comparables o hasta más bajas que buses convencionales a motor diésel.

El impacto ambiental en términos de gases nocivos es significativo y alto en términos de GEI por la red eléctrica casi $100 \%$ en base de energía renovable. Los BEBs ya son una tecnología comprobada con costos de inversión y de mantenimiento en descenso. En ciudades chinas se utilizan flotas grandes de BEBs en circunstancias similares a la GAM y múltiples ciudades en China ya están en proceso de reemplazar la totalidad de la flota por este tipo de buses (en Shenzhen ya 100\% de la flota es eléctrica) ( $L u$, Xue, \& Zhou, 2018).

Sin embargo, para poder llegar a esta realidad en Costa Rica, aún se deben tomar ciertas medidas para aligerar la transición que se enumerarán a continuación.

\section{Tarifa de Electricidad para el transporte}

Actualmente no hay una tarifa específica para el transporte eléctrico. La tarifa que se utilizaría incluye el costo por potencia - y esto crítico en todos cálculos financieros. También es crítico para determinar el tipo de BEBs por comprar (carga lenta nocturna o carga intermedia en horas valle). Se recomienda definir lo antes posible una política de precios de energía para vehículos eléctricos especialmente vehículos eléctricos de transporte público - ya que el impacto no es solo en el área ambiental. El impacto social y el posicionamiento de Costa Rica en la región con una tecnología innovadora, justifica tener precios bajos para la energía eléctrica en este ámbito.

El factor de costo que incide fuertemente en el caso de transporte eléctrico es el costo por potencia. Se recomienda revisar este costo para el sector de transporte, especialmente para BEBs que se cargan en horas valle y horas nocturnas.

\section{Política de Inversión en Cargadores}

El propietario (y en general operador) de la infraestructura de cargadores puede ser el operador de transporte, la empresa eléctrica o una empresa tercera. Los tres sistemas existen en diferentes ciudades. Se recomienda ver la opción en la que la empresa eléctrica invierta en infraestructura de cargadores en coordinación con los operadores, que deben ser consultados para determinar la ubicación y la capacidad de los cargadores.

La provisión de la infraestructura de recarga para autobuses no es tan compleja como para el transporte particular, pero debe ser coordinada muy bien con los transportistas; pues los tipos de cargadores dependen de los tipos de buses, su forma de uso y la ubicación de 
los cargadores. Es crítico tomar estos factores en cuenta para garantizar una operación óptima.

A nivel político, se debería considerar la posibilidad de que la inversión en cargadores y su mantenimiento y operación se recupere a través de la factura eléctrica de todos los ciudadanos en vez de cargar todo el costo de inversión al transportista. Esto haría el transporte eléctrico económicamente más factible. Este subsidio cruzado se justifica, ya que los altos subsidios impositivos a vehículos eléctricos particulares que favorecen básicamente los sectores con un poder adquisitivo alto. Se considera socialmente justo de financiar parcialmente la transición del transporte público a un transporte electrificado a través de la factura eléctrica común. Esta política es seguida en varios países que subsidian la instalación de cargadores eléctricos.

\section{Estrategia de Reducción de Riesgos}

Existen variaciones bastante elevadas en rendimientos y costos (p.ej. de mantenimiento) de BEBs entre diferentes ciudades y existe todavía cierta inseguridad por la vida útil de baterías y sus costos en el futuro.

Factores importantes que influyen la rentabilidad de los buses eléctricos son los precios futuros del diésel y los precios de la electricidad. Sin embargo, el riesgo y la percepción del riesgo de BEBs por parte de los transportistas en Costa Rica influyen significativamente en la transición. Por ende, instrumentos de mitigación de riesgo e incentivos financieros son necesarios.

Una forma para reducir los riesgos es, adquiriendo una flota lo suficientemente grande (de mínimo 50 unidades). Eso permite de reducir considerablemente los costos de inversión y asegurar un mantenimiento base, ya que se recibe apoyo de los fabricantes para el entrenamiento del personal. Además, permite asegurar un stock de repuestos que disminuye el tiempo de espera de buses en caso de fallas.

Ciudades que han invertido en flotas piloto han ganado cierta experiencia con estos buses, pero esa estrategia ha resultado siempre en costos elevados y una baja disponibilidad de los buses por falta de experiencia en mantenimiento, menor soporte de fabricantes y por falta de repuestos. BEBs son fabricados básicamente por empresas chinas y muchos de los repuestos no están disponibles en el mercado local por lo cual es de suma importancia de tener una flota homogénea suficientemente grande.

\section{Regulaciones y Políticas de Transporte}

Políticas que se consideran importantes para fomentar transporte público eléctrico incluyen:

- Tener una tarifa de transporte al usuario que no aumente por ser un bus eléctrico. Si se realiza un aumento de la tarifa debido al transporte eléctrico resulta un cambio modal hacía transporte particular y además afecta la capa social más vulnerable. El cambio modal resulta en más emisiones y disminuye por ende el efecto de electrificar el transporte. Eventuales riesgos o costos mayores por el transporte eléctrico deben ser compensados por otros mecanismos financieros o por subsidios justificados por el impacto ambiental del transporte eléctrico.

- Diferenciar la vida útil máxima permitida por tecnología de bus teniendo el BEB una vida mayor que buses convencionales. Técnicamente se justifica por tener menos partes movibles y por ende menos fatiga en partes críticas y además por no tener un motor fósil que con el tiempo ya no cumple con los estándares más exigentes de contaminación.

- Implementar una política que no permita la entrada de buses fósiles a partes centrales de la GAM por tener en esas áreas elevadas índices de contaminación. Como mínimo se debería favorecer en licitaciones para nuevas rutas o renovaciones de rutas el uso de buses eléctricos.

- El panorama para una transición hacia el transporte público eléctrico en el GAM se muestra favorable, sin embargo, aún se debe trabajar en varias políticas que catalicen la transición. La variable que presenta menos riesgos es la de buses de 12 metros con baterías moderadas que se recarguen en los planteles durante la noche.

- Actualmente la Firma Grütter Consulting está trabajando en conjunto con el ICE un modelo de negocios para poder traer 100 buses eléctricos para el transporte público en el próximo año y darlos en leasing. El proyecto se visualiza como económica- y financieramente viable. También la disposición del mismo sector de empresarios en general se percibe como positiva, ya que no tienen que incurrir en gastos adicionales ni asumir ellos el riesgo con una tecnología aún no puesta en prueba. Si adicionalmente el país logra implementar algunas de las políticas previamente descritas, se puede decir efectivamente que hay un camino trazado para la adopción de esta tecnología. 


\section{REFERENCIAS}

ARESEP (Autoridad Reguladora de Servicios Públicos). (2018, 11 de setiembre). Pliego tarifario de autobús. Recuperado de https://aresep.go.cr/tarifas/tarifas-vigentes/2529-tarifas-de-autobus-11-setiembre-2018

Asian Development Bank (2018, noviembre) Sustainable Urban Transport Buses, Low Carbon Buses in the People's Republic of China.
Gobierno Londres. (2017, 14 de julio). Cleaner buses. Recuperado de https://www.london.gov.uk/whatwe-do/environment/pollution-and-air-quality/ cleaner-buses

Lu, L., Xue, L., \& Zhou, W. (2018, April 4). How Did Shenzhen, China Build World's Largest Electric Bus Fleet? Recuperado de https://www.wri.org/blog/2018/04/how-did-shenzhen-china-build-world-s-largest-electric-bus-fleet

Riteve. (2017). Revisión Técnica Vehicular 2017, Reporte Anual. Coordinación: Jennifer Hidalgo V. Recuperado de https://www.rtv.co.cr/wp-content/uploads/ AnuarioRiteve2017.pdf 